\title{
Elevated Neutrophil-to-Lymphocyte Ratio in Squamous Cell Carcinoma of Larynx Compared to Benign and Precancerous Laryngeal Lesions
}

\author{
Rauf Oguzhan Kum ${ }^{1 *}$, Muge Ozcan ${ }^{1}$, Deniz Baklaci ${ }^{1}$, Nurcan Yurtsever Kum ${ }^{1}$, \\ Yavuz Fuat Yılmaz ${ }^{1}$, Volkan Gungor ${ }^{1}$, Adnan Unal ${ }^{2}$
}

\begin{abstract}
Background: Laryngeal carcinogenesis is a multifactorial process that has not been fully elucidated. Despite extensive research, reliable markers with diagnostic and prognostic value are still lacking. It was recently reported that an elevated preoperative neutrophil-to-lymphocyte ratio (NLR) may correlate with an increased risk of recurrence, tumor aggressiveness and poorer prognosis in various malignancies. The aim of this study was to examine whether NLR could be used as an inflammatory marker to differentiate laryngeal squamous cell carcinoma (LSCC) patients from benign laryngeal lesion (BLL) and precancerous laryngeal lesion (PLL) patients. Materials and Methods: This retrospective study was performed on 209 patients admitted to a tertiary referral center with laryngeal lesions and undergoing biopsies to establish their histopathological diagnosis. We reviewed the patient files for their clinical, histopathological and laboratory data. The patients were divided into three groups according to their histopathological findings, as BLL, PLL and LSCC groups. The patients in the PLL group were also divided into three subgroups as mild, moderate and severe dysplasia/ carcinoma in situ (CIS) subgroups. The groups were compared for NLR and the other laboratory data. Results: The mean NLRs of the BLL, PLL and the LSCC groups were $2.12 \pm 0.86,2.32 \pm 0.68$ and 3.46 \pm 1.51 , respectively, and the difference was statistically significant $(p=0.001)$. The mean NLRs of the patients with PLL and LSCC were significantly higher than the patients with $B L L$ ( $p=0.031$ and $p=0.001$, respectively). The mean NLRs were similar among mild dysplasia, moderate dysplasia and severe dysplasia / CIS groups (p>0.05). Conclusions: To our knowledge, this is the first study investigating NLR in BLL, PLL and LSCC. NLR is an inexpensive, reproducible and widely available blood test, and could be a useful inflammatory marker to differentiate LSCC from BLL and PLL.
\end{abstract}

Keywords: Squamous cell carcinoma of larynx - preneoplasia - neutrophil-lymphocyte ratio

Asian Pac J Cancer Prev, 15 (17), 7351-7355

\section{Introduction}

Laryngeal squamous cell carcinomas (LSCC) are the most frequently seen cancers among head and neck squamous cell carcinomas (HNSCC), and they constitute 1-2\% of all malignancies worldwide (Sadri et al., 2006). Laryngeal carcinogenesis is a multifactorial process that has not been fully elucidated. LSCC develops as a result of structural and cytological alterations in the laryngeal squamous epithelium (Marcos et al., 2011). Transformation of precancerous laryngeal lesions (PLL), which include various degrees of dysplasia and carcinoma in situ (CIS), to invasive cancer is not infrequent (Ferlito et al., 2012). Currently, there is no sufficient evidence for the use of biomarkers in predicting the future behavior of laryngeal dysplastic lesions in the clinical practice (Nankivell et al., 2011). Despite extensive research, reliable markers with diagnostic and prognostic value are still lacking.

Today, it is supposed that the inflammatory reaction of the host against tumor, and the inflammation caused indirectly by tumor increase angiogenesis, damage DNA, and facilitate proliferation of tumor and its metastasis by preventing apoptosis (Jaiswal et al., 2000; Coussens and Werb, 2002). Recent studies have shown correlations between the degree of systemic inflammatory response and outcomes in various tumors (Roxburgh and McMillan, 2010). Neutrophil-to-lymphocyte ratio (NLR) is a validated marker of systemic inflammation (Ozdemir et al., 2014). Published data suggest that an elevated preoperative NLR may correlate with an increased risk of recurrence, tumor aggressiveness, poorer prognosis, propensity for metastasis and death in various malignancies (Karaman et al., 2013; Kacan et al., 2014; Kemal et al., 2014). 
The aim of this study was to examine whether NLR could be used as an inflammatory marker to differentiate LSCC patients from benign laryngeal lesion (BLL) and PLL patients. To our knowledge, this is the first study that has investigated the relationship of an elevated preoperative NLR in BLL, PLL and LSCC patients.

\section{Materials and Methods}

This retrospective study was performed on 209 patients who admitted to a tertiary referral center with laryngeal lesions between May 2010 and May 2014, and had biopsies to establish their histopathological diagnosis. Our study was approved by the local ethics committee, and conducted in accordance with the ethical principles of Declaration of Helsinki.

We reviewed the patients' files for their clinical, histopathological and laboratory data. Only subjects with available preoperative complete blood counts (CBC) with differential were included in the study. Preoperative NLR, MPV, platelet distribution width (PDW), white blood cell (WBC), platelet, neutrophil, and lymphocyte counts, demographic characteristics and histopathological data of the patients were analyzed. The patients who had any inflammatory, autoimmune, acute or chronic infectious diseases, hematological disorders, history of corticosteroid therapy or chronic renal insufficiency were excluded.

All WBC and differential counts were obtained within the week prior to biopsy, before the patients received any treatment. NLR was calculated as a simple ratio between the absolute neutrophil and the absolute lymphocyte counts. An automated blood cell counter (Beckman Coulter analyzer, California, USA) was used for CBC measurements. $\mathrm{CBC}$ analyses were performed within 2 hours after blood sampling.

The patients were divided into three groups according to their histopathological findings, as BLL, PLL and LSCC groups. The patients in the PLL group were also divided into three subgroups as mild, moderate and severe

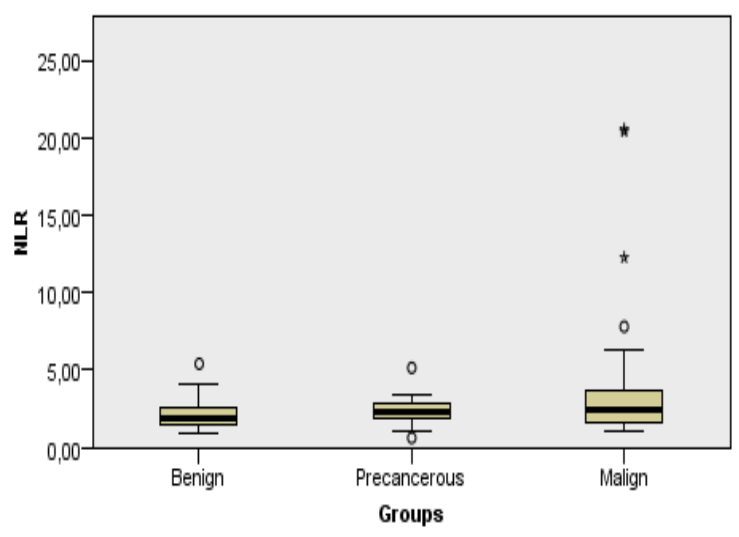

Figure 1. Mean Neutrophil-to-Lymphocyte Ratios in Benign Laryngeal Lesion, Precancerous Laryngeal Lesion and Laryngeal Squamous Cell Carcinoma Groups.

Table 1. The Demographic Characteristics and the Variables Studied in Benign, Precancerous and Malignant Laryngeal Lesion (SCC) Groups

\begin{tabular}{|c|c|c|c|c|}
\hline & $\begin{array}{c}\text { 1Benign }(n=80) \\
\text { Mean } \pm S D\end{array}$ & $\begin{array}{c}\text { 2Precancerous }(n=63) \\
\text { Mean } \pm S D\end{array}$ & $\begin{array}{c}3 \mathrm{SCC}(\mathrm{n}=66) \\
\text { Mean } \pm \mathrm{SD}\end{array}$ & $\mathrm{p}$ \\
\hline Age (years) & $47.33 \pm 12.63$ & $57.14 \pm 9.88$ & $63.45 \pm 9.57$ & \\
\hline MPV (fl) & $9.91 \pm 1.72$ & $9.99 \pm 1.29$ & $9.78 \pm 1.55$ & 0.745 \\
\hline PDW (fl) & $14.38 \pm 2.38$ & $13.66 \pm 2.37$ & $14.35 \pm 2.58$ & 0.160 \\
\hline WBC (103/U) & $8.00 \pm 1.95$ & $7.89 \pm 1.87$ & $8.39 \pm 2.06$ & 0.321 \\
\hline Platelet (103/U) & $262.33 \pm 66.85$ & $237.34 \pm 58.97$ & $254.10 \pm 73.88$ & 0.085 \\
\hline Neutrophil (103/U) & $4.77 \pm 1.57$ & $5.15 \pm 1.60$ & $5.34 \pm 1.77$ & 0.106 \\
\hline Lymphocyte (103/U) & $2.41 \pm 0.70$ & $2.26 \pm 0.51$ & $2.06 \pm 0.81$ & $0.013 * *$ \\
\hline \multirow[t]{2}{*}{ NLR } & $2.12 \pm 0.86$ & $2.32 \pm 0.68$ & $3.46 \pm 1.51$ & $\begin{aligned} 0.001 * *{ }^{1-2} \mathrm{p} & =0.031 * * \\
{ }^{2-3} \mathrm{p} & =0.068 \\
{ }^{1-3} \mathrm{p} & =0.001 * *\end{aligned}$ \\
\hline & $\mathrm{n}(\%)$ & $\mathrm{n}(\%)$ & $\mathrm{n}(\%)$ & Total n (\%) \\
\hline Gender & $63(78.7 \%)$ & $54(85.7 \%)$ & $64(97.0 \%)$ & $181(86.6 \%)$ \\
\hline Female & $17(21.3 \%)$ & $9(14.3 \%)$ & $2(3.0 \%)$ & $28(13.4 \%)$ \\
\hline
\end{tabular}

*p<0.05**SD: Standard deviation, MPV: Mean platelet volume, PDW: Platelet distribution width, WBC: White blood cell, NLR: Neutrophil-to-lymphocyte ratio, SCC: Squamous cell carcinoma

Table 2. Mean Analysis for Neutrophil-to-Lymphocyte Ratio and Other Complete Blood Count Parameters of Precancerous Laryngeal Lesion Subgroups

\begin{tabular}{lccc}
\hline & $\begin{array}{c}\text { Mild dysplasia } \\
(\mathrm{n}=23) \\
\text { Mean } \pm \text { SD }\end{array}$ & $\begin{array}{c}\text { Moderate dysplasia } \\
(\mathrm{n}=24) \\
\text { Mean } \pm \text { SD }\end{array}$ & $\begin{array}{c}\text { Severe dysplasia }(\mathrm{n}=11) / \\
\text { CIS }(\mathrm{n}=5)(\mathrm{n}=16) \\
\text { Mean } \pm \text { SD }\end{array}$ \\
\hline WBC $(103 / \mathrm{U})$ & $7.89 \pm 2.54$ & $7.83 \pm 1.35$ & $7.99 \pm 1.51$ \\
Platelet $(103 / \mathrm{U})$ & $225.78 \pm 53.09$ & $234.20 \pm 44.95$ & $258.68 \pm 80.22$ \\
Neutrophil $(103 / \mathrm{U})$ & $4.92 \pm 1.83$ & $5.28 \pm 1.53$ & $5.27 \pm 1.41$ \\
Lymphocyte $(103 / \mathrm{U})$ & $2.17 \pm 0.68$ & $2.38 \pm 0.42$ & $2.20 \pm 0.29$ \\
NLR & $2.34 \pm 0.81$ & $2.25 \pm 0.62$ & 0.965 \\
\hline
\end{tabular}

*p<0.05**SD: Standard deviation, MPV: Mean platelet volume, PDW: Platelet distribution width, WBC: White blood cell, NLR: Neutrophil-to-lymphocyte ratio, CIS: Carcinoma in situ 
dysplasia/CIS subgroups (Gale et al., 2005). The patients in the BLL group had histopathological diagnoses of inflammation, vocal nodule or vocal polyp. All of the patients in LSCC group had histopathological diagnosis of squamous cell carcinoma. The groups were compared for NLR, MPV, PDW, WBC, and platelet, neutrophil and lymphocyte counts.

Statistical Package for Social Sciences (SPSS) for Windows 21.0 software was used for the statistical analyses. The descriptive data were presented as mean \pm standard deviation. For comparison of the quantitative data that did not show a normal distribution among 3 or more groups, the Kruskal Wallis test was used. Mann Whitney U test was used for two-group comparisons of data with an abnormal distribution. The significance was set at $\mathrm{p}<0.05$.

\section{Results}

A total of 209 patients, $181(86.6 \%)$ males and 28 (13.4\%) females were included in the study. BLL group consisted of 80 patients, $63(78.7 \%)$ males and $17(21.3 \%)$ females. PLL group consisted of $54(85.7 \%)$ males and $9(14.3 \%)$ females, and a total of 63 patients, and LSCC group consisted of 66 patients comprising 64 (97.0\%) males and $2(3.0 \%)$ females. The mean ages of BLL, PLL and LSCC groups were $47.33 \pm 12.63,57.14 \pm 9.88$ and $63.45 \pm 9.57$ years, respectively (Table 1 ).

The histopathological diagnosis was inflammation in 23 , vocal nodule in 24, and vocal polyp in 33 patients in BLL group. In PLL group, 23 patients had mild dysplasia, 24 had moderate dysplasia, 11 had severe dysplasia, and 5 patients had CIS.

The mean lymphocyte counts of the BLL, PLL and the LSCC groups were $2.41 \pm 0.7010^{3} / \mathrm{U}, 2.26 \pm 0.51103 / \mathrm{U}$ and $2.06 \pm 0.8110^{3} / \mathrm{U}$, respectively, and the difference was statistically significant $(\mathrm{p}=0.013)$ (Table 1$)$.

The mean NLR of the BLL, PLL and the LSCC groups were $2.12 \pm 0.86,2.32 \pm 0.68$ and $3.46 \pm 1.51$, respectively, and the difference was statistically significant $(\mathrm{p}=0.001)$ (Table 1). The paired comparisons made to determine the group that caused the difference revealed that the mean NLR of the patients with PLL and LSCC were significantly higher than the patients with BLL ( $\mathrm{p}=0.031$ and $\mathrm{p}=0.001$, respectively). However, the difference between the mean NLR of PLL and LSCC groups was not significant $(\mathrm{p}=0.068)($ Table 1) (Figure 1).

MPV, PDW, and WBC, neutrophil and platelet counts were similar among the BLL, PLL and LSCC groups ( $\mathrm{p}>0.05)$ (Table 1).

NLR, and WBC, neutrophil, lymphocyte and platelet counts were similar among mild dysplasia, moderate dysplasia and severe dysplasia / CIS groups $(\mathrm{p}>0.05)$ (Table 2).

\section{Discussion}

HNSCC is the sixth most commonly seen cancer in the world, and more than $90 \%$ of the tumors occur in larynx, oral cavity and pharynx (Haddad and Shin, 2008; Jemal et al., 2008). Adequate diagnosis and treatment of the precursor lesions is needed to improve survival. A laryngeal mucosal precancerous lesion is defined as an altered epithelium with an increased likelihood of progression to LSCC (Gale et al., 2005).

Inflammation can be caused by infection, autoimmune diseases, malignant and benign tumors and other pathologies, and results in infiltration of the inflammatory cells at specific sites in the body. Inflammation is thought to contribute to the development and progression of various cancers, and inflammation in the body can be detected in the peripheral blood.

Recent studies have confirmed a link between the inflammatory microenvironment of a tumor, and systemic responses induced by the tumor. For many types of cancer, lymphocytopenia indicates a generalized state of immunodepression (Wenger et al., 1999), and survival appears to be adversely influenced by depressed immune function. The number of CD4+ helper lymphocytes may decrease, and CD8+ suppressor lymphocytes may increase due to disturbed inflammatory response, and immunosuppression may result (Menges et al., 1999). The increase in the numbers of neutrophils and / or decreased numbers of lymphocytes may suppress lymphokine-activated killer cells. These may be the possible mechanisms for decreased survival in cancer patients (Teramukai et al., 2009).

The presence of an elevated preoperative NLR has been validated as a marker of inflammation, and shown to have prognostic significance for a number of tumors (Jung et al., 2011; Idowu et al., 2012; Liu et al., 2013; Unal et al., 2013), including HNSCC (Trellakis et al., 2011; Millrud et al., 2012; Perisanidis et al., 2013; Rassouli et al., 2013; Tsai et al., 2013). It is considered as a marker of systemic inflammation which may predict worse prognosis by driving cancer cell proliferation through increasing the availability of growth factors, angiogenic factors and other pro-neoplastic signaling molecules (Hanahan and Weinberg, 2011). NLR may represent the two opposing inflammatory and immune pathways that exist together in cancer patients (Azab et al., 2012).

In our study, we have hypothesized that NLR reflects the systemic inflammatory response created by the laryngeal lesions, and could be a used as an inflammatory marker to differentiate patients with LSCC from the ones with benign and precancerous laryngeal lesions. To our knowledge, no studies up to date have investigated the significance of NLR in patients with precancerous and benign laryngeal lesions, and LSCC.

Recently, Hanahan et al. reported the role of uncontrolled inflammation in driving tumor proliferation, suggesting a possible reason behind baseline NLR and its prognostic utility (Hanahan and Weinberg, 2011). Seretis et al. reported that the mean NLR of the patients with papillary thyroid carcinoma (PTC) was significantly higher than the patients with benign goiter. The authors concluded that NLR could potentially be used as a biomarker for detecting incidental small PTC in an apparently benign goiter. They also reported that NLR might be a promising serum biomarker for diagnosis and prognostication of PTC (Seretis et al., 2013). In another study, Tsai et al. reported an increase in NLR in parallel 
with the advancement of clinical stage in the patients with oral cavity cancer, and in contrast, a reduction in the lymphocyte count (Tsai et al., 2013). Similarly, pretreatment NLR was found as a strong prognostic factor in nasopharyngeal carcinoma patients (An et al., 2011).

Rassouli et al. reported that NLR was an easily obtainable pretreatment inflammatory marker, and could be used as predictor of survival and recurrence in HNSCC patients (Rassouli et al., 2013). Another study reported that systemic inflammation as well as the numbers of monocytes, neutrophils and total leukocytes increased, and NLR enhanced in HNSCC patients (Millrud et al., 2012). Trellakis et al. reported significantly higher NLR in HNSCC patients compared to controls (Trellakis et al., 2011). However, in all of the aforementioned studies, HNSCC patients were studied without taking the origin of the tumor into account, and the significance of NLR was not studied in LSCC in comparison with benign and precancerous laryngeal lesions. In our study, we aimed to examine whether NLR could be a useful inflammatory marker to differentiate LSCC patients from BLL and PLL patients. We found that NLR was the lowest in BLL, higher in PLL and the highest in LSCC. This finding suggests that NLR may be used as a marker for malignant transformation of benign laryngeal lesions, and possibly, recurrence of the laryngeal cancer.

In conclusion, our study is the first one that investigated NLR in BLL, PLL and LSCC. NLR is an inexpensive, reproducible and widely available blood test, and it could be a useful inflammatory marker to differentiate LSCC patients from BLL and PLL patients. The limitations of our study are its retrospective and single-center study design, the lack of evaluation of tumor-associated neutrophils and lymphocytes, and lack of prospective clinical follow up of our patients. Because this area of research is a novel field, prospective, multicenter studies on a larger numbers of patients are needed to comprehensively identify the patients with inflammatory response at high risk for poor outcomes.

\section{References}

An X, Ding PR, Wang FH, Jiang WQ, Li YH (2011). Elevated neutrophil to lymphocyte ratio predicts poor prognosis in nasopharyngeal carcinoma. Tumour Biol, 32, 317-24.

Azab B, Bhatt VR, Phookan J, et al (2012). Usefulness of the neutrophil-to-lymphocyte ratio in predicting short-and longterm mortality in breast cancer patients. Ann Surg Oncol, 19, 217-24.

Coussens LM, Werb Z (2002). Inflammation and cancer. Nature, 420, 860-7.

Ferlito A, Devaney KO, Woolgar JA, et al (2012). Squamous epithelial changes of the larynx: diagnosis and therapy. Head Neck, 34, 1810-6.

Gale N, Pilch BZ, Sidransky D, Westra W, Califano J (2005). Tumours of the hypopharynx, larynx and trachea (epithelial precursor lesions).In 'World Health Organization Classification of Tumours: Pathology and Genetics of Head and Neck Tumours', L. Barnes, J. W. Eveson, P. Reichart and D. Sidranksy. IARC Press, Lyon, France., 140-3.

Haddad RI, Shin DM (2008). Recent advances in head and neck cancer. N Engl J Med, 359, 1143-54.

Hanahan D, Weinberg RA (2011). Hallmarks of cancer: the next generation. Cell, 144, 646-74.

Idowu OK, Ding Q, Taktak AF, Chandrasekar CR, Yin Q (2012). Clinical implication of pretreatment neutrophil to lymphocyte ratio in soft tissue sarcoma. Biomarkers, 17, 539-44.

Jaiswal M, LaRusso NF, Burgart LJ, Gores GJ (2000). Inflammatory cytokines induce DNA damage and inhibit DNA repair in cholangiocarcinoma cells by a nitric oxidedependent mechanism. Cancer Res, 60, 184-90.

Jemal A, Siegel R, Ward E, et al (2008). Cancer statistics, 2008. CA Cancer J Clin, 58, 71-96.

Jung MR, Park YK, Jeong O, et al (2011). Elevated preoperative neutrophil to lymphocyte ratio predicts poor survival following resection in late stage gastric cancer.J Surg Oncol, 104, 504-10.

Kacan T, Babacan NA, Seker M, et al (2014). Could the neutrophil to lymphocyte ratio be a poor prognostic factor for non small cell lung cancers? Asian Pac J Cancer Prev, 15, 2089-94.

Karaman H, Karaman A, Erden A, et al (2013). Relationship between colonic polyp type and the neutrophil/ lymphocyte ratio as a biomarker. Asian Pac J Cancer Prev, 14, 3159-61.

Kemal Y, Yucel I, Ekiz K, et al (2014). Elevated serum neutrophil to lymphocyte and platelet to lymphocyte ratios could be useful in lung cancer diagnosis. Asian Pac J Cancer Prev, 15, 2651-4.

Liu CL, Lee JJ, Liu TP, et al (2013). Blood neutrophil-tolymphocyte ratio correlates with tumor size in patients with differentiated thyroid cancer. J Surg Oncol, 107, 493-7.

Marcos CA, Alonso-Guervos M, Prado NR, et al (2011). Genetic model of transformation and neoplastic progression in laryngeal epithelium. Head Neck, 33, 216-24.

Menges T, Engel J, Welters I, et al (1999). Changes in blood lymphocyte populations after multiple trauma: association with posttraumatic complications. Crit Care Med, 27, 733-40.

Millrud CR, Kvarnhammar AM, Uddman R, et al (2012). The activation pattern of blood leukocytes in head and neck squamous cell carcinoma is correlated to survival. PLoS One, 7, e51120.

Nankivell P, Weller M, McConkey C, Paleri V, Mehanna H (2011). Biomarkers in laryngeal dysplasia: a systematic review. Head Neck, 33, 1170-6.

Ozdemir Y, Akin ML, Sucullu I, Balta AZ, Yucel E (2014). Pretreatment neutrophil/lymphocyte ratio as a prognostic aid in colorectal cancer. Asian Pac J Cancer Prev, 15, 2647-50.

Perisanidis C, Kornek G, Poschl PW, et al (2013). High neutrophil-to-lymphocyte ratio is an independent marker of poor disease-specific survival in patients with oral cancer. Med Oncol, 30, 1-8.

Rassouli A, Saliba J, Castano R, Hier M, Zeitouni AG (2013). Systemic inflammatory markers as independent prognosticators of head and neck squamous cell carcinoma. Head Neck, [Epub ahead of print].

Roxburgh CS, McMillan DC (2010). Role of systemic inflammatory response in predicting survival in patients with primary operable cancer. Future Oncology, 6, 149-63.

Sadri M, McMahon J, Parker A (2006). Laryngeal dysplasia: aetiology and molecular biology. J Laryngol Otol, 120, 170-7.

Seretis C, Gourgiotis S, Gemenetzis G, et al (2013). The significance of neutrophil/lymphocyte ratio as a possible marker of underlying papillary microcarcinomas in thyroidal goiters: a pilot study. Am J Surg, 205, 691-6.

Teramukai S, Kitano T, Kishida Y, et al (2009). Pretreatment neutrophil count as an independent prognostic factor in advanced non-small-cell lung cancer: an analysis of Japan 
Multinational Trial Organisation LC00-03. Eur J Cancer, 45, 1950-8.

Trellakis S, Bruderek K, Dumitru CA, et al (2011). Polymorphonuclear granulocytes in human head and neck cancer: enhanced inflammatory activity, modulation by cancer cells and expansion in advanced disease. Int J Cancer, 129, 2183-93.

Tsai YD, Wang CP, Chen CY, et al (2013). Pretreatment circulating monocyte count associated with poor prognosis in patients with oral cavity cancer. Head Neck, 36, 947-53

Unal D, Eroglu C, Kurtul N, Oguz A, Tasdemir A (2013). Are neutrophil/lymphocyte and platelet/lymphocyte rates in patients with non-small cell lung cancer associated with treatment response and prognosis? Asian Pac J Cancer Prev, 14, 5237-42.

Wenger FA, Jacobi CA, Zieren J, et al (1999). Tumor size and lymph-node status in pancreatic carcinoma-is there a correlation to the preoperative immune function? Langenbecks Arch Surg, 384, 473-8. 